\title{
Treat to target deep remission with mucosal healing: new goals of medical therapy in ulcerative colitis
}

Volume I Issue 2 - 2014

\begin{abstract}
Editorial
One of the most successful and beneficial results of basic immunologic research has been the development and application of anti-tumor necrosis alpha (anti-TNF $\alpha$ ) monoclonal antibodies in rheumatoid and psoriatic arthritis and inflammatory bowel disease (IBD). The results in idiopathic IBD, i.e. ulcerative colitis and Crohn's disease, have been the change in treatment goals. It is no longer a clinical remission that is achieved, i.e. reduction and control of symptoms but now "deep remission" is sought, i.e. endoscopic and histologic remission.
\end{abstract}

The paradigm of successful IBD treatment now requires "deep remission". The difficulty ("conundrum") is with the clinically well patient who lacks complete deep remission yet has difficulty in accepting the burden of additional drug therapy with greater risks and costs. However, the data is compelling that when deep remission is achieved less complication, surgery, hospitalization and tumor development can be expected. ${ }^{1-5}$ Nevertheless, $40 \%$ of patients may lose this response with continued treatment beyond 1 to 2 years. ${ }^{6}$

\section{Factors attributed to this loss of response include}

i. Increasing immunogenicity of these agents to produce anti-drug antibodies (ADA) which accelerate their clearance.

ii. Failure to monitor and adjust ongoing medical therapy for mucosal healing which is considered an excellent surrogate marker for a continued benefit in addition to fecal calprotectin and lactoferrin biomarkers.

iii. Superimposition of infection often opportunistic or malignancy (melanoma, lymphoma, etc).

iv. Loss of response or drug intolerance.

The drive to achieve mucosal healing $(\mathrm{MH})$ with its apparent benefits is the true goal of successful therapy but the "target" of the "treat to target" impetus remains problematic. Firstly, a precise definition of $\mathrm{MH}$ remains elusive although it usually requires complete absence of inflammatory and ulcerating disease on endoscopy and histology. Yet there are no parameters of the degree of MH needed nor is there recognition of levels of mucosal improvement following the use of biologic agents. ${ }^{8}$ Secondly, is endoscopy necessary when there is a clear correlation of $\mathrm{MH}$ with therapeutic levels of the biologic agent (e.g. infliximab) and normal biomarkers? ${ }^{9}$ Fecal markers such as fecal calprotectin (FCP) or lactoferrin (LF) correlate nicely with the severity of inflammation and often reliably reflect the endoscopic response to treatment. A normal FCP is often acceptable as a marker for successful MH. Similarly fecal lactoferrin is a sensitive and specific index of chronic inflammation often predictive of relapse. ${ }^{10}$ Actually there is no clear definition of MH in ulcerative colitis (UC). ${ }^{11}$ Equally unclear is the question of risks of continued or increased therapy necessary to improve the degree of $\mathrm{MH} .{ }^{12}$

\author{
Seymour Katz \\ New York University School of Medicine, USA
}

Correspondence: Seymour Katz, New York University School of Medicine, North Shore, University Hospital-Long Island Jewish Health System, Manhasset, St Francis Hospital, Roslyn, New York, USA, Emal seymourkatz.md@gmail.com

Received: July 17,2014 | Published: May 17, 2014

Can anti-TNF therapy be optimized early in the treatment regimen since $40 \%$ of primary responders will fail particularly with longer duration disease, prior smoking, or a genetic predisposition? Monitoring drug levels (infliximab or adalimumab) to increase dose, shorten dosing intervals or detecting anti-drug antibody would necessitate switching to a different drug formulation or one with a different mechanism. These drug and antibody levels are touted as necessary management tools particularly with the better antibody assay permitting drug and antibody levels in the same sample. ${ }^{13}$ In a secondary loss of response seen in 23 to $46 \%$ of patients on anti-TNF therapy longer than 12 months, monitoring such levels has become the considered standard of care.

Other therapies in non-responders include anti-cytokine antibodies to interleukin (IL)-6R, IL-13, IL-12/IL-23 or those anti-inflammatory agents that regulate T cells, Smad7 antisense, JAK inhibition, or stimulation of Toll-like receptor 9. More recent progress had been shown with blocking T-cell egress into diseased tissue with antiintegrins $\alpha 4 \beta 7$ specific to the GI tract or targeting mucosal vascular address in cell adhesion molecule 1 (MAdCAM-1). ${ }^{14}$ Manipulating the microbiome shows promising strategies with non-absorbable antibiotics, probiotics, prebiotics, diet manipulations (e.g. FODMAP) or combinations of these agents. Fecal microbiota transplantation is an enticing option but controlled clinical data is wanting. ${ }^{15}$ Low vitamin $\mathrm{D}$ levels may alter responses to anti-TNF therapy (if levels are less than $27 \mathrm{ng} / \mathrm{mL}$ ) causing earlier cessation of anti-TNF therapeutic program. ${ }^{16}$

Despite a recent report that $\mathrm{MH}$ was not predictive of a sustained remission, ${ }^{17} \mathrm{MH}$ has assumed the position as the most reproducible and accepted marker of treatment success and is a predictor of the future course ${ }^{18}$ provided adjustments in therapy ("treat to target") are made along the way to achieve this goal.

\section{Acknowledgements}

The author is grateful to Huafeng Shen, MD for technical assistance.

\section{Conflicts of interest}

Author declares there are no conflicts of interest. 


\section{Funding}

None.

\section{References}

1. D'Haens G, Noman M, Baert F, et al Endoscopic healing after infliximab treatment for Crohn's disease provides a longer time to relapse Gastroenterology. 2002;122(Suppl 4):A-618.

2. Froslie KF, Jahnsen J, Moum BA, et al. Mucosal healing in inflammatory bowel disease:results from a Norwegian population-based cohort. Gastroenterology. 2007;133(2):412-422.

3. Schnitzler F, Fidder H, Ferrante M, et al. Mucosal healing predicts long-term outcome of maintenance therapy with infliximab in Crohn's disease. Inflamm Bowel Dis. 2009;15(9):1295-1301.

4. Peyrin-Biroulet L, Ferrante M, Magro F, et al. Results from the 2nd Scientific Workshop of the ECCO. I:Impact of mucosal healing on the course of inflammatory bowel disease. J Crohns Colitis. 2011;5(5):477483.

5. Colombel JF, Louis E, Peyrin-Biroulet L, et al. Deep remission:a new concept? Dig Dis. 2012;30(Suppl 3):107-111.

6. Van Assche G. Optimizing biologic therapy for treatment of inflammatory bowel disease. Gastroenterol Hepatol (N Y). 2013;9(7):462-464.

7. Altwegg R, Vincent T. TNF blocking therapies and immunomonitoring in patients with inflammatory bowel disease. Mediators Inflamm. 2014;10.1155/2014/172821.

8. Mazzuoli S, Guglielmi FW, Antonelli E, et al. (2013) Definition and evaluation of mucosal healing in clinical practice. Dig Liver Dis. 2013;45(12):969-977.

9. Paul S, Del Tedesco E, Marotte H, et al. Therapeutic drug monitoring of infliximab and mucosal healing in inflammatory bowel disease:a prospective study. Inflamm Bowel Dis. 2013;19(12):2568-2576.
10. Stragier E, Van Assche G. The use of fecal calprotectin and lactoferrin in patients with IBD. Review. Acta Gastroenterol Belg. 2013;76(3):322328.

11. Zallot C, Peyrin-Biroulet L. Deep remission in inflammatory bowel disease: looking beyond symptoms. Curr Gastroenterol Rep. 2013;15(3):315.

12. Rogler G, Vavricka S, Schoepfer A, et al. Mucosal healing and deep remission: what does it mean? World J Gastroenterol. 2013;19(43):75527560 .

13. Ben-Horin S, Kopylov U, Chowers Y. Optimizing anti-TNF treatments in inflammatory bowel disease. Autoimmun Rev. 2014;13(1):24-30.

14. Neurath MF. New targets for mucosal healing and therapy in inflammatory bowel diseases. Mucosal Immunol. 2014;7(1):6-19.

15. D'Haens GR, Sartor RB, Silverberg MS, et al. Future directions in inflammatory bowel disease management. $J$ Crohns Colitis. 2014;pii:S1873-S9946(14):00094-00104.

16. Zator ZA, Cantu SM, Konijeti GG, et al. Pretreatment 25hydroxyvitamin $\mathrm{D}$ levels and durability of anti-tumor necrosis factor- $\alpha$ therapy in inflammatory bowel diseases. JPEN J Parenter Enteral Nutr. 2014;38(3):385-391.

17. Farkas K, Lakatos PL, Szucs M, et al. Frequency and prognostic role of mucosal healing in patients with Crohn's disease and ulcerative colitis after one-year of biological therapy. World $J$ Gastroenterol. 2014;20(11):2995-3001.

18. Bouguen G, Levesque BG, Pola S, et al. Endoscopic assessment and treating to target increase the likelihood of mucosal healing in patients with crohn's disease. Clin Gastroenterol Hepatol. 2013;pii:S1542S3565(13):01742-01744. 